\title{
mFOLFOXIRI with or without bevacizumab for conversion therapy of RAS/BRAF/PIK3CA mutant unresectable colorectal liver metastases: the FORBES non-randomized phase II trial
}

\author{
Cailu Shen ${ }^{1,2 \#}$, Huabin $\mathrm{Hu}^{1,2 \#}$, Yue Cai ${ }^{1,2}$, Jiayu Ling ${ }^{1,2}$, Jianwei Zhang ${ }^{1,2}$, Zehua Wu ${ }^{1,2}$, Xiaoyu Xie ${ }^{1,2}$, \\ Meijin Huang ${ }^{2,3}$, Hui Wang ${ }^{2,3}$, Liang Kang ${ }^{2,3}$, Ping Lan ${ }^{2,3}$, Xiaojian Wu ${ }^{2,3}$, Guangjian Liu ${ }^{4}$, Yunle Wan ${ }^{5}$, \\ Zhiyang Zhou, Yan Huang ${ }^{7}$, Fangqian $\mathrm{Li}^{6}$, Huaiming Wang ${ }^{2,3}$, Tenghui $\mathrm{Ma}^{2,3}$, Shuangling Luo ${ }^{2,3}$, \\ Yonghua $\mathrm{Cai}^{2,3}$, Lishuo $\mathrm{Shi}^{8}$, Yanhong Deng ${ }^{1,2}$
}

${ }^{1}$ Department of Medical Oncology, The Sixth Affiliated Hospital, Sun Yat-sen University, Guangzhou, China; ${ }^{2}$ Guangdong Institute of Gastroenterology, Guangdong Provincial Key Laboratory of Colorectal and Pelvic Floor Diseases, Guangzhou, China; ${ }^{3}$ Department of Colorectal Surgery, The Sixth Affiliated Hospital, Sun Yat-sen University, Guangzhou, China; ${ }^{4}$ Department of Medical Ultrasonics, The Sixth Affiliated Hospital, Sun Yat-sen University, Guangzhou, China; ${ }^{5}$ Department of Hepatobiliary Surgery, The Sixth Affiliated Hospital, Sun Yat-sen University, Guangzhou, China; ${ }^{6}$ Department of Radiology, The Sixth Affiliated Hospital, Sun Yat-sen University, Guangzhou, China; ${ }^{7}$ Department of Pathology, The Sixth Affiliated Hospital, Sun Yat-sen University, Guangzhou, China; ${ }^{8}$ Department of Clinical Research Center, The Sixth Affiliated Hospital, Sun Yat-sen University, Guangzhou, China

Contributions: (I) Conception and design: C Shen, H Hu, L Shi, Y Deng; (II) Administrative support: Y Deng; (III) Provision of study materials or patients: M Huang, H Wang, L Kang, P Lan, X Wu, G Liu, Y Wan, Z Zhou, Y Huang, F Li, HM Wang, T Ma, S Luo, YH Cai; (IV) Collection and assembly of data: C Shen, H Hu, Y Cai, J Ling, J Zhang, Z Wu, X Xie, Y Deng; (V) Data analysis and interpretation: C Shen, H Hu, Y Cai, J Ling, J Zhang, Z Wu, X Xie, L Shi, Y Deng; (VI) Manuscript writing: All authors; (VII) Final approval of manuscript: All authors.

\#These authors contributed equally to this work.

Correspondence to: Yanhong Deng. Department of Medical Oncology, The Sixth Affiliated Hospital, Sun Yat-sen University, Yuan Cun Er Heng Road, Guangzhou 510655, China. Email: dengyanh@mail.sysu.edu.cn.

Background: The aim of this non-randomized single-center phase II trial was to prospectively assess the clinical efficacy of triplet chemotherapy with modified 5 -fluorouracil, folinic acid, oxaliplatin, and irinotecan (mFOLFOXIRI) plus bevacizumab as conversion therapy for initially unresectable rat sarcoma viral oncogene homolog (RAS)/v-raf murine sarcoma viral oncogene homolog B1 (BRAF)/phosphatidylinositol-3 kinase catalytic alpha (PIK3CA) mutant colorectal liver-limited metastases (CRLMs).

Methods: Patients with $R A S / B R A F / P I K 3 C A$ mutant initially unresectable CRLMs were recruited at a ratio of 2:1 to receive mFOLFOXIRI plus bevacizumab (experimental group) or mFOLFOXIRI alone (control group). The rate of patients attaining no evidence of disease (NED) was the primary endpoint. The secondary endpoints included objective response rate (ORR), depth of tumor response (DpR), secondary resection rate, progression-free survival (PFS), overall survival (OS), and safety.

Results: The rate of NED achieved was $40.7 \%$ and $30.8 \%$, respectively, in the experimental $(\mathrm{n}=54)$ and control groups $(n=26)$; the adjusted odds ratio was 4.519 [95\% confidence interval (CI): 1.247-16.375, $\mathrm{P}=0.022]$. The ORR was $77.4 \%$ in the experimental group and $60.0 \%$ in the control group $(\mathrm{P}=0.112)$. The median DpR was significantly greater in the experimental group ( $45.6 \%$ vs. $34.9 \%, \mathrm{P}=0.041$ ). The median PFS was 12.6 months in the experimental group and 9.1 months in the control group [adjusted hazard ratio (HR): $0.584,95 \% \mathrm{CI}: 0.304-1.121, \mathrm{P}=0.106]$. Median OS was prolonged in the experimental group compared with the control group (42.6 vs. 35.3 months, adjusted HR: 0.443, 95\% CI: 0.195-1.006, P=0.052). Thirty patients $(55.6 \%)$ in the experimental group and $16(61.5 \%)$ in the control group experienced grade $3 / 4$ adverse events.

Conclusions: We observed that the combination of mFOLFOXIRI and bevacizumab increased the rate of clinical NED and showed a trend toward improved survival compared with mFOLFOXIRI alone. This could represent a conversion therapy option for fit patients with initially unresectable $R A S / B R A F / P I K 3 C A$ 
mutant CRLMs.

Keywords: Conversion therapy; colorectal liver-limited metastases (CRLMs); mutant; bevacizumab; triplet chemotherapy

Submitted Nov 12, 2021. Accepted for publication Jan 13, 2022.

doi: 10.21037/atm-21-6731

View this article at: https://dx.doi.org/10.21037/atm-21-6731

\section{Introduction}

Around $50 \%$ of patients with colorectal cancer develop liver metastases (LMs) during the course of their disease (1). These patients are considered a special subset, considering the potentially curative option provided by the multidisciplinary treatment approach for those with colorectal liver-limited metastases (CRLMs) (2). The benefits of liver resection are well established. However, only $10-20 \%$ of cases are considered suitable for resection due to the multifocal disease, inadequate functional liver reserve, and poor anatomic localization (3).

The recommended front-line treatment for patients with unresectable metastatic colorectal cancer (mCRC) is traditional chemotherapy plus a biologically targeted agent, either anti-vascular endothelial growth factor monoclonal antibody (bevacizumab) or anti-epidermal growth factor receptor monoclonal antibody (cetuximab or panitumumab) (4). Upfront active systemic chemotherapy with a conversion therapy intent often comprises doublet chemotherapy, that is, 5-fluorouracil, leucovorin, and oxaliplatin (FOLFOX) or irinotecan (FOLFIRI), combined with bevacizumab or cetuximab. Several studies on this chemotherapy have been shown to downsize LMs, making secondary resection possible $(5,6)$. Nevertheless, decisionmaking involving the resection of LMs is affected by several factors, including technical resectability, risk factors for early recurrence, general operability, and patient's preference (7). Recently, "toolbox" instruments for local ablative treatment (LAT), including metastasectomy, ablation, embolization, and stereotactic body radiation therapy (SBRT) techniques, have been applied to maximize the possibility of removing all visible LMs and attain no evidence of disease (NED) (8). The addition of LAT to chemotherapy has been associated with a significant improvement in survival $(9,10)$. Clinical guidelines recommend using systemic therapy in combination with LAT strategy to achieve tumor clearance in non-surgical patients (7).

Recent findings have revealed that triplet chemotherapy
(FOLFOXIRI) has better efficacy than doublet regimens (FOLFOX/FOLFIRI) in the metastatic setting, including patients with CRLMs $(11,12)$. The OLIVIA study compared bevacizumab with triplet versus doublet, and the results showed that bevacizumab with triplet had a better objective response rate (ORR), an R0 resection rate, and progression-free survival (PFS) (13). Studies with direct comparison of efficacy in patients with initially unresectable CRLMs treated with FOLFOXIRI alone and FOLFOXIRI plus bevacizumab have not been conducted. The clinical benefit of adding bevacizumab to the FOLFOXIRI regimen as conversion therapy in this setting remains unclear, especially in tumors harbor rat sarcoma viral oncogene homolog $(R A S) / \mathrm{v}$-raf murine sarcoma viral oncogene homolog B1 $(B R A F) /$ phosphatidylinositol-3 kinase catalytic alpha (PIK3CA) mutation, which together account for almost $60 \%$ of mCRC cases $(14,15)$. While FOLFOXIRI regimen has a quite high response, it also resulted in a markedly increased of grade 2 or 3 peripheral neurotoxicity ( $0 \%$ vs. $19 \%, \mathrm{P}<0.001)$ and grade 3 or 4 neutropenia ( $28 \%$ vs. $50 \%, \mathrm{P}<0.001)$ when compared to the FOLFIRI regimen in the phase III GONO trial (11). To improve the tolerability of FOLFOXIRI regimen, we proposed a modified FOLFOXIRI (mFOLFOXIRI) regimen in which the dosage of 5-fluorouracil and irinotecan are reduced. We therefore conducted this FORBES study comparing mFOLFOXIRI alone or in combination with bevacizumab as conversion therapy in this setting.

We present the following article in accordance with the TREND reporting checklist (available at https://atm. amegroups.com/article/view/10.21037/atm-21-6731/rc).

\section{Methods}

\section{Patients}

Patients had histologically confirmed colorectal adenocarcinoma with initially unresectable liver-limited metastases were eligible for inclusion. Unresectable LMs 
were defined as meeting $\geq 1$ of the following criteria: no possibility of upfront R0 or R1 resection of all LM lesions; inadequate $(<30 \%)$ future liver remnant after resection; and all hepatic liver veins, both hepatic arteries, or both portal vein branches infiltrated. LMs were assessed using contrast-enhanced computed tomography (CT) or magnetic resonance imaging (MRI). Unresectability was evaluated by a local multidisciplinary team (MDT). Other criteria for eligibility were detection of $R A S$ (codons 12, 13, 59, 61, 117 , and 146 of $K R A S$ and NRAS) or BRAF (codon 600) or PIK3CA (exons 9 and 20) status, and at least 1 of these genes carrying a mutation; primary tumor deemed resectable; age between 18 and 70 years and Eastern Cooperative Oncology Group performance status (PS) score $\leq 1$ (patients between 71 and 75 years of age were eligible if their PS score was 0 ); adequate organ function at the start of study treatment; and life expectancy of $\geq 3$ months. The main exclusion criteria were as follows: prior adjuvant therapy for colorectal cancer completed with an interval of $<6$ months before enrollment; peripheral neuropathy of grade $>1$ according to the National Cancer Institute Common Terminology Criteria for Adverse Events (CTCAE), version 4.0; and definite extrahepatic metastases.

\section{Study design and treatment}

This was a prospective non-randomized phase II study conducted at a single colorectal cancer center. Patients were recruited to receive mFOLFOXIRI plus bevacizumab (experimental group) or mFOLFOXIRI alone (control group) at a ratio of 2:1 based on their choice (Table S1). mFOLFOXIRI chemotherapy regimen (day 1: irinotecan $165 \mathrm{mg} / \mathrm{m}^{2}$, oxaliplatin $85 \mathrm{mg} / \mathrm{m}^{2}$, and folinic acid $400 \mathrm{mg} / \mathrm{m}^{2}$, followed by a $46-\mathrm{h}$ continuous infusion of 5 -fluorouracil $2,800 \mathrm{mg} / \mathrm{m}^{2}$ on day 1 , all given intravenously) was administered to the control group patients. Patients in the experimental group received bevacizumab $5 \mathrm{mg} / \mathrm{kg}$ body weight intravenous infusion on day 1 , followed by the same regimen of mFOLFOXIRI (16). A treatment cycle was defined as 14 days (2 weeks) in each group. Patients continued treatment until disease progression, or they had unacceptable toxicity, or a maximum of 12 cycles was reached. In the experimental group, the administration of bevacizumab was discontinued 6-8 weeks prior to all surgical resections, while mFOLFOXIRI chemotherapy was continued during this time interval. Bevacizumab was discontinued 2 weeks before thermal ablation procedure. The strategy to obtain adequate LATs was decided on by a local MDT consisting of at least 1 colorectal surgeon, 1 oncologist, 2 liver surgeons and 2 radiologists, and was based on local experience, tumor characteristics, and patient preference. A 2-stage approach was used to attain NED for LMs. Subsequently, following NED, patients were advised to continue the same treatment regimen for a total of 12 cycles (6 months); more than 1 study agent could be interrupted or discontinued if the patients experienced intolerance or any grade $3 / 4$ toxicity. For patients who experienced grade $\geq 2$ leukopenia and/or neutropenia after the previous treatment cycle, granulocyte colony-stimulating factor for prophylaxis was allowed. Finally, subsequent therapy was provided to patients who either failed the conversion treatment or experienced progressive disease according to the oncologist's choice. The study was conducted in accordance with the Declaration of Helsinki (as revised in 2013). This study was approved by ethics committee of The Sixth Affiliated Hospital, Sun Yat-sen University (No. 2014ZSLYEC-024) and informed consent was taken from all individual participants. The study protocol was registered in the ClinicalTrials.gov (identifier: NCT02350530).

\section{Study endpoints and tumor assessments}

In this trial, the rate of patients attaining NED was the primary endpoint. Secondary endpoints included ORR, depth of tumor response ( $\mathrm{DpR})$, overall resection rate (R0/R1/R2), macroscopically complete resection rate, $\mathrm{R} 0$ resection rate, PFS, overall survival (OS), and safety. Eligible patients receiving at least 1 dose of treatment were evaluable for efficacy and safety. Tumor responses were assessed through radiographic measurements based on abdominopelvic and thoracic contrast-enhanced CT or abdominal MRI at baseline and after every 4 treatment cycles using Response Evaluation Criteria In Solid Tumors (RECIST) version 1.1. For patients undergoing surgical resection, margin status (R0/R1/R2) was evaluated by the histopathological assessment of resected metastases, together with the operation note. R0 resection was defined as no residual tumor status, $\mathrm{R} 1$ resection indicated a microscopically positive resection $(<1 \mathrm{~mm}$ from the resection margin), while $\mathrm{R} 2$ resection was defined as a gross residual tumor (17). A tumor was considered as complete thermal ablation if no nodular or irregular enhancement adjacent to the ablation zone was visible on contrastenhanced CT or MRI at the 4-week follow up posttreatment (8).

The percentage of patients who obtained complete 


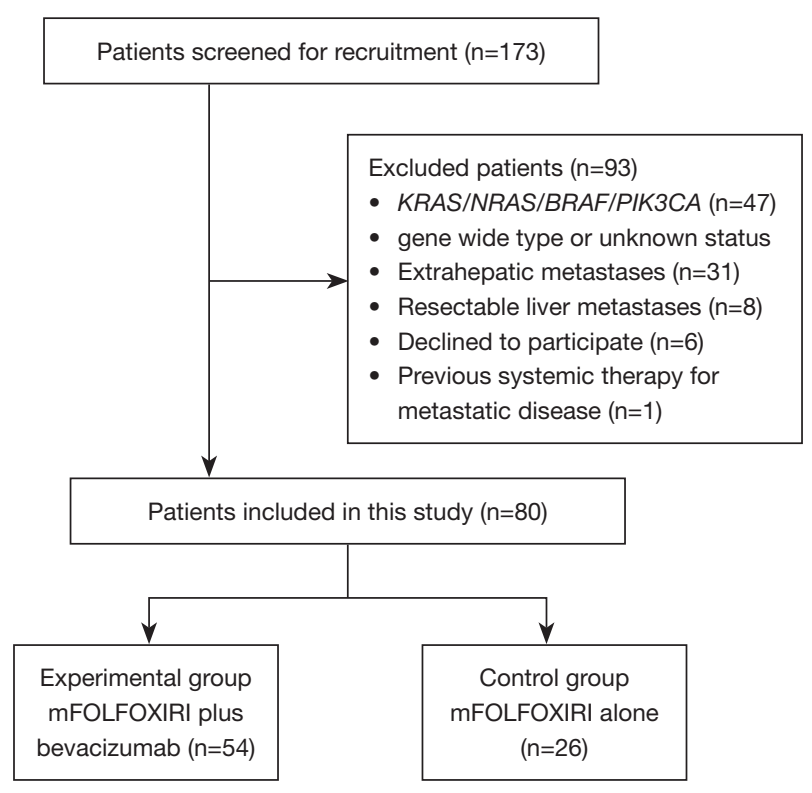

Figure 1 Trial profile. FOLFOXIRI, 5-fluorouracil, folinic acid, oxaliplatin, and irinotecan; mFOLFOXIRI, modified FOLFOXIRI.

remission, R0 resection, or macroscopically complete ablation of all visible tumor masses was defined as the rate of NED achieved. The proportion of patients with a complete response (CR) or partial response (PR) achieved after initiation of the study treatment was defined as ORR. DpR was calculated as the maximum change in the sum of the longest diameters of the target lesions compared with baseline. PFS was calculated from the date of registration to the date of first documented tumor progression or death resulting from any cause, whichever occurred first. Patients who were alive without experiencing progression were censored for PFS at the last on-study tumor assessment date. OS was measured from the date of registration to the date of death resulting from any cause. Patients were censored for OS at the last follow up if they were still alive or lost to follow up. Clinical NED achieved and tumor response were assessed by both the radiologist and oncologist. The safety data were analyzed descriptively. Adverse events (AEs) were assessed on the basis of the nature, frequency, and severity according to CTCAE, version 4.0.

\section{Statistical analysis}

Sample size estimation was performed using PASS software, version 15.0 (NCSS, LLC, East Kaysville, UT, USA). We postulated that $15 \%$ of patients would achieve NED status in the control group and $45 \%$ in the experimental group. Seventy-eight patients in total were required to recruited at a ratio of 2:1; 52 for the experimental group and 26 for the control group. The statistical analysis used the Z-test (pooled), with two-sided $\alpha=0.05$ at a power of $80 \%$.

KRAS and NRAS mutations were grouped in a single category: $R A S$ mutation. Data were analyzed using MannWhitney $U$ test, $\chi^{2}$ test (Pearson's $\chi^{2}$ test, correction for continuity), or Fisher's exact test, where appropriate. The disease response was described via waterfall plots, separated by treatment groups. The analysis of PFS and OS was performed using the Kaplan-Meier method and log-rank test. The median follow-up was calculated according to the inverse Kaplan-Meier method. Due to the observational nature of this study, multivariate analyses were conducted with logistic regression models or Cox regression models to adjust for baseline differences. Odds ratios, hazard ratios (HRs), and 95\% confidence intervals (CIs) were estimated using corresponding regression models. All statistical tests were two-sided, and the level of significance was set at $\mathrm{P}<0.05$. Statistical analyses were performed using SPSS, version 26.0 (IBM, Armonk, NY, USA).

\section{Results}

\section{Patient characteristics and treatment}

A total of 173 consecutive patients were screened for recruitment between January 2015 and October 2020. Of these, 93 were excluded for reasons outlined in the patient flowchart (Figure 1). Finally, 80 enrolled patients were analyzed in our study. Patient- and tumor-related characteristics were similar between the experimental group ( $n=54)$ and control group $(n=26)$, but a significantly higher proportion of patients in the experimental group had $>10$ LMs compared with those in the control group (44.4\% vs. $15.4 \%, \mathrm{P}=0.031$ ) (Table 1).

The median number of chemotherapy cycles of mFOLFOXIRI was six in the control group (range, 1-9) and eight in the experimental group (range, 2-12). The mean dose intensity for single cytotoxic drugs within the control group was slightly higher than the experimental group. Treatment exposure is described in Table S2. The cutoff date for survival data was March 31, 2021, with a median follow-up time of 30.0 months (95\% CI: $26.3-$ 33.7 months) in the experimental group compared with 49.0 months (95\% CI: 40.6-57.4 months) in the control 
Table 1 Baseline demographic and clinical characteristics of patients

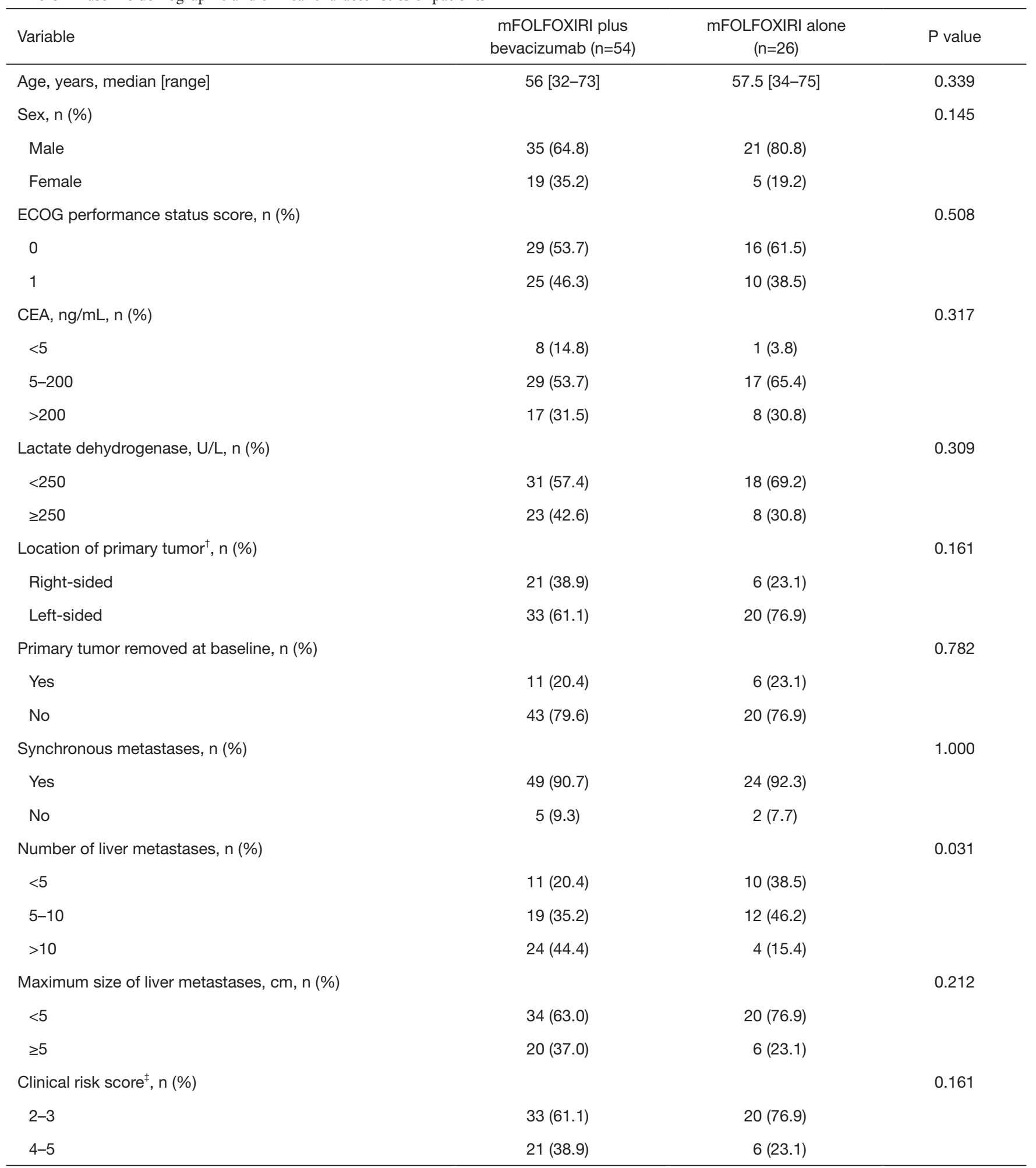

Table 1 (continued) 
Table 1 (continued)

\begin{tabular}{|c|c|c|c|}
\hline Variable & $\begin{array}{l}\text { mFOLFOXIRI plus } \\
\text { bevacizumab }(n=54)\end{array}$ & $\begin{array}{l}\text { mFOLFOXIRI alone } \\
\qquad(n=26)\end{array}$ & $P$ value \\
\hline \multicolumn{4}{|l|}{ Reasons for unresectability, n (\%) } \\
\hline No upfront R0 or R1 resection of hepatic lesions possible & $47(87.0)$ & $21(80.8)$ & 0.462 \\
\hline \multicolumn{4}{|l|}{ Mutated gene, n (\%) } \\
\hline RAS (KRAS or NRAS) & $48(88.9)$ & $20(76.9)$ & 0.160 \\
\hline$B R A F^{\Uparrow}$ & $4(7.4)$ & $5(19.2)$ & 0.142 \\
\hline Adjuvant therapy, n (\%) & & & 0.547 \\
\hline None & $51(94.4)$ & $26(100.0)$ & \\
\hline Treated & $3(5.6)$ & $0(0.0)$ & \\
\hline
\end{tabular}

${ }^{\dagger}$, right-sided included tumors from cecum to transverse colon; left-sided represented tumors from splenic flexure to rectum; ${ }^{\ddagger}$, clinical risk factors included lymphatic metastases of primary cancer, simultaneous metastasis or interval $<12$ months from primary tumor resection to metastasis, CEA $>200 \mathrm{ng} / \mathrm{mL}$, number of liver metastasis $>1$, and maximum size of liver metastasis $>5 \mathrm{~cm}$. Each risk factor was 1 point. There were no patients with a score of 0 or 1 in each group; ", one patient in the bevacizumab group harbored the G466E mutation and one harbored the F468_G469delinsSS mutation in the control group, and the others had V600E mutation. ECOG, Eastern Cooperative Oncology Group; FOLFOXIRI, 5-fluorouracil, folinic acid, oxaliplatin, and irinotecan; mFOLFOXIRI, modified FOLFOXIRI; CEA, carcinoembryonic antigen.

group. Thirty-four patients $(79.1 \%)$ in the experimental group and 12 patients $(60.0 \%)$ in the control group with primary tumor in situ had resection of the primary tumor during the study period. Sixteen patients $(61.5 \%)$ in the control group and 32 patients $(59.3 \%)$ in the experimental group underwent LATs. A total of 55 patients experienced disease progression. The second-line treatment regimens are shown in Table $\mathrm{S} 3$.

\section{Efficacy}

Of the 80 patients evaluated for efficacy, NED achieved was noted in 30 patients (37.5\%, 95\% CI: $27.6-48.5 \%$ ), and shown a trend favoring the experimental group (22 of 54 patients, $40.7 \%$; $95 \%$ CI: $28.7-54.0 \%$ ) compared with the control group (8 of 26 patients, $30.8 \%$; $95 \%$ CI: $16.5-50.0 \%)$ on the basis of the directionality of the odds ratio (1.547, 95\% CI: $0.572-4.180$ ), but not significantly so
$(\mathrm{P}=0.390)$ (Table 2). The analysis after adjusting for baseline imbalance variables (number of LMs) revealed that the odds ratio for NED associated with the experimental group was 4.519 (95\% CI: 1.247-16.375; $\mathrm{P}=0.022$ ) (Table 3). Among these 30 patients attaining NED, 1 achieved clinical CR, 5 underwent R0 hepatic resection, 6 had R0 hepatic resection plus complete thermal ablation, 1 underwent R0 resection plus complete thermal ablation and SBRT, and another 17 had complete thermal ablation. The median duration of NED achieved was 11.5 (95\% CI: 8.0-15.0) months in the experimental group; the median duration of NED achieved was not calculated in the control group owing to small number of patients. Nineteen patients (5 in the control group and 14 in the experimental group) underwent resection of LMs; the overall resection rate (R0/R1/R2) was slightly higher in the experimental group ( $25.9 \%$ vs. $19.2 \%, \mathrm{P}=0.510)$. An overall response was observed in $77.4 \%$ of the patients in the experimental group 
Table 2 Results for efficacy end points

\begin{tabular}{|c|c|c|c|}
\hline Variable & $\begin{array}{l}\text { mFOLFOXIRI plus } \\
\text { bevacizumab }(n=54)\end{array}$ & $\begin{array}{l}\text { mFOLFOXIRI alone } \\
\qquad(\mathrm{n}=26)\end{array}$ & $P$ value \\
\hline \multirow[t]{2}{*}{ NED achieved, n (\%) } & $22(40.7)^{\dagger}$ & $8(30.8)$ & 0.390 \\
\hline & & & $0.022^{*}$ \\
\hline Overall resection rate, $\mathrm{n}(\%)$ & $14(25.9)$ & $5(19.2)$ & 0.510 \\
\hline $\mathrm{R} 0$ resection plus complete thermal ablation & $6(11.1)$ & $0(0.0)$ & 0.170 \\
\hline $\mathrm{R} 0$ resection plus complete thermal ablation and SBRT & $1(1.9)$ & $0(0.0)$ & 1.000 \\
\hline $\mathrm{R} 2$ resection & $3(5.6)$ & $3(11.5)$ & 0.384 \\
\hline ORR (CR + PR), n (\%) & $41(77.4)$ & $15(60.0)$ & 0.112 \\
\hline $\mathrm{CR}$ & $1(1.9)$ & $0(0.0)$ & \\
\hline PR & $40(75.5)$ & $15(60.0)$ & \\
\hline SD & $11(20.8)$ & $9(36.0)$ & \\
\hline PD & $1(1.9)$ & $1(4.0)$ & \\
\hline DpR, median (IQR), \% & $45.6(31.0-64.9)$ & $34.9(14.2-46.0)$ & 0.041 \\
\hline \multicolumn{4}{|l|}{ Progression-free survival, months } \\
\hline
\end{tabular}

${ }^{\dagger}$, one patient achieved cCR; ", one patient in each group could not be evaluated because of radiographic assessment was not performed; *, $\mathrm{P}$ values were adjusted for the number of liver metastases of patients. $\mathrm{Cl}$, confidence interval; CR, complete response; DpR, depth of response; IQR, interquartile range; FOLFOXIRI, 5-fluorouracil, folinic acid, oxaliplatin, and irinotecan; mFOLFOXIRI, modified FOLFOXIRI; NED, no evidence of disease; ORR, objective response rate; PR, partial response; PD, progressive disease; SD, stable disease; SBRT, stereotactic body radiation therapy; TACE, transarterial chemoembolisation; cCR, clinical complete response.

compared with $60.0 \%$ in the control group (odds ratio, 2.28 ; 95\% CI: 0.82-6.36; $\mathrm{P}=0.112$ ). Additionally, the median DpR was $45.6 \%$ [interquartile range (IQR), 31.0-64.9\%] with bevacizumab versus $34.9 \%$ (IQR, 14.2-46.0\%) without bevacizumab $(\mathrm{P}=0.041)$. Figure 2 presents the maximum percent changes in tumor size in the two groups. Examples of radiological imaging before and after treatment in the two groups are shown in Figure S1.

The median PFS was 12.6 months in the experimental group (95\% CI: 11.2-14.0 months) and 9.1 months (95\% CI: 6.3-11.9 months) in the control group. The HR for progression or death associated with the experimental group was 0.711 (95\% CI: $0.389-1.300 ; \mathrm{P}=0.264)$. The analysis after adjusting baseline imbalance variables revealed that the experimental group was associated with a $42 \%$ reduced risk of progression compared with the control group (95\% CI: 0.304-1.121; $\mathrm{P}=0.106$ ) (Figure 3A). The median PFS significantly improved in patients attaining NED (19.9 vs. 
Table 3 Multivariable logistic regression analysis evaluating possible risk factors associated with NED

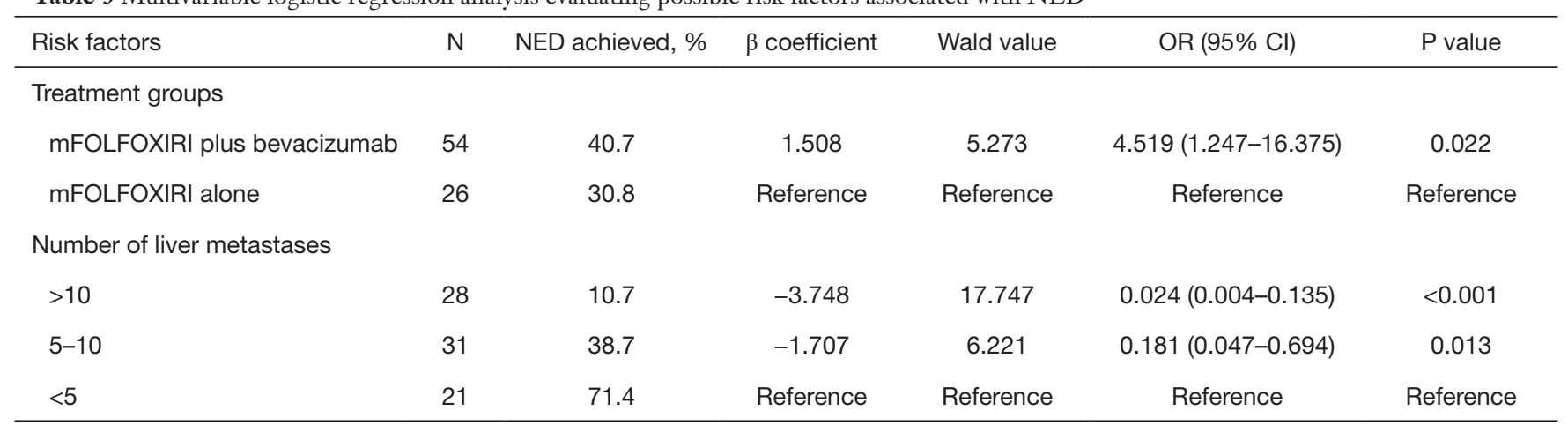

$\mathrm{CI}$, confidence interval; FOLFOXIRI, 5-fluorouracil, folinic acid, oxaliplatin, and irinotecan; mFOLFOXIRI, modified FOLFOXIRI; NED, no evidence of disease; OR, odds ratio.

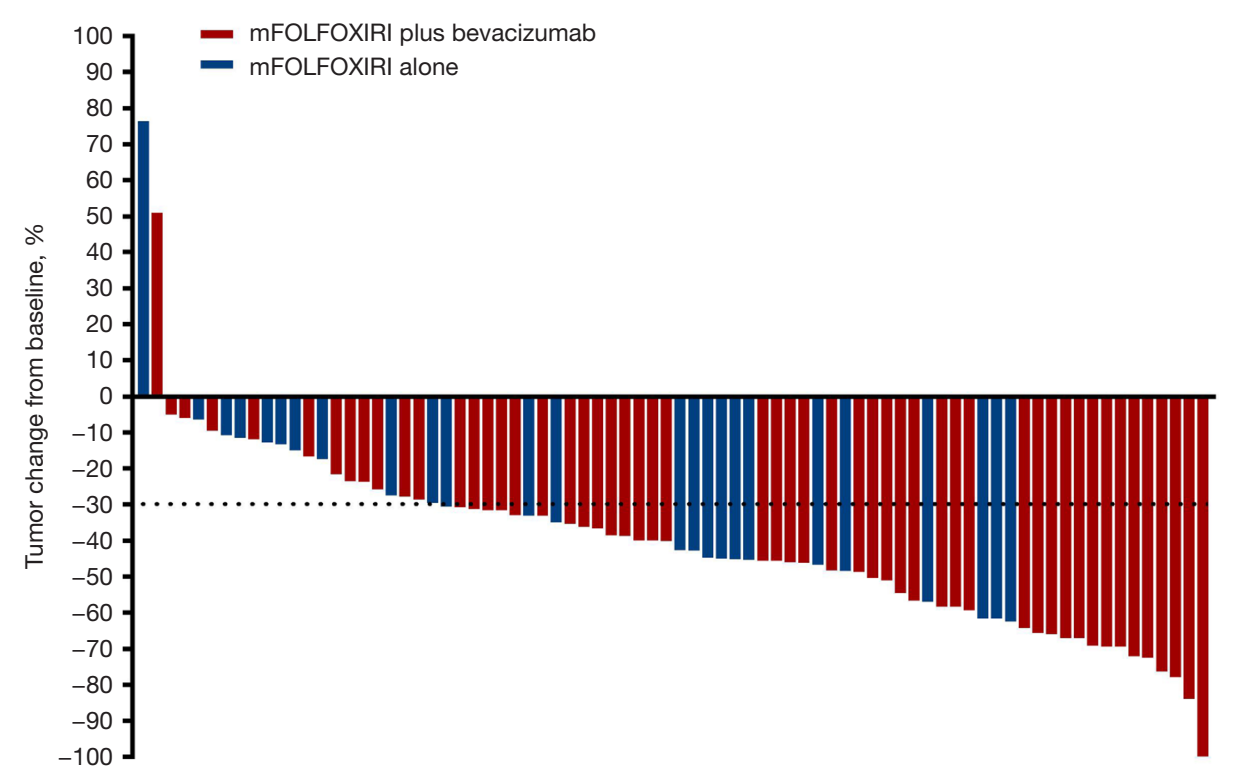

Figure 2 Waterfall plot of maximum percent change in tumor size from baseline as measured, according to RECIST version 1.1 . FOLFOXIRI, 5-fluorouracil, folinic acid, oxaliplatin, and irinotecan; mFOLFOXIRI, modified FOLFOXIRI.

8.9 months; HR, 0.195; 95\% CI: 0.101-0.375; P<0.001). A significant survival benefit was observed in patients who attained NED compared with those not in the experimental group $(\mathrm{P}<0.001)$ (Figure $3 B)$ and the control group $(\mathrm{P}=0.001)$. The median OS for patients in the experimental group was 42.6 months compared with 35.3 months for patients in the control group. The HR for death associated with the experimental group was 0.719 (95\% CI: 0.352-1.466; $\mathrm{P}=0.362)$. The experimental group was associated with a $56 \%$ reduced risk of death compared with the control group after adjusting baseline imbalance variables (95\% CI: $0.195-$ 1.006; $\mathrm{P}=0.052$ ) (Figure 3C). In all patients, the median
OS was significantly prolonged in patients who attained NED compared with those who did not (not reached $v s$. 20.7 months; HR, 0.147; 95\% CI: 0.056-0.385; $\mathrm{P}<0.001$ ). Consistent results were also observed in terms of significant benefits in patients who attained NED compared with those not in the experimental group $(\mathrm{P}=0.001)$ (Figure $3 D)$ and the control group $(\mathrm{P}=0.002)$.

\section{Safety and toxicity}

Regarding safety, no case of grade 5 and unexpected toxicities was reported in either group. Toxicity events 
A

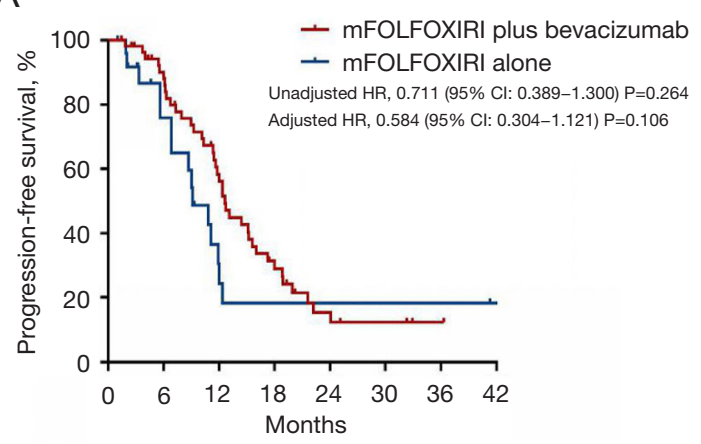

C

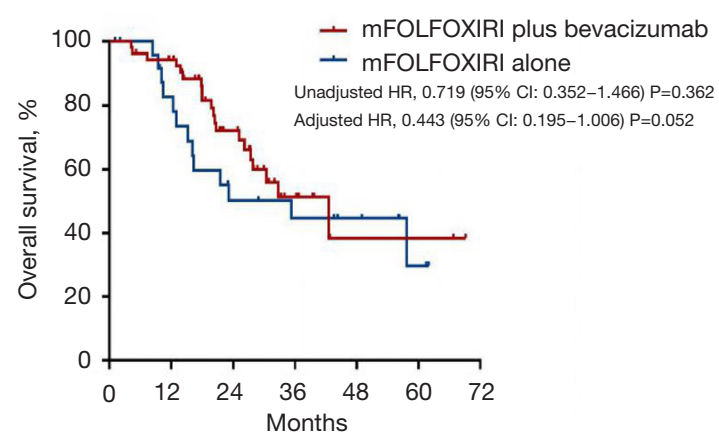

B

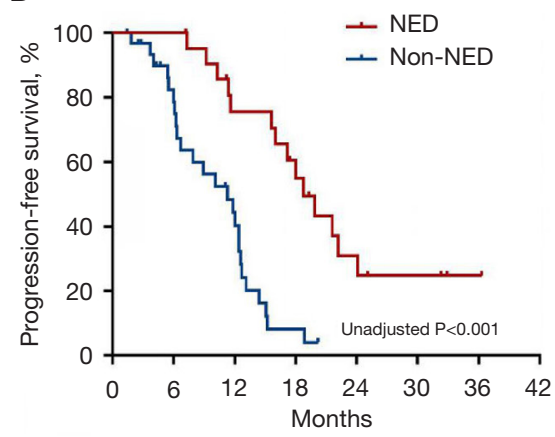

D

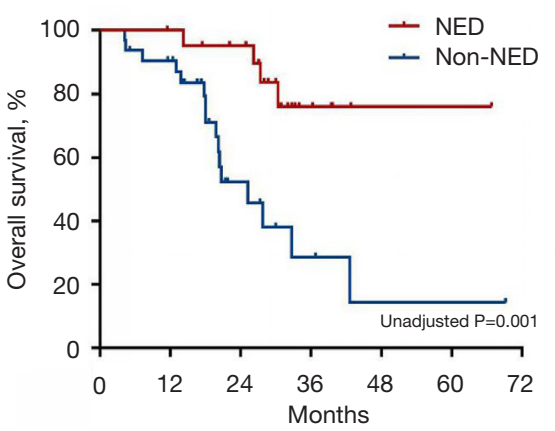

Figure 3 Kaplan-Meier curves of progression-free and overall survival. (A) Progression-free survival according to treatment groups; (B) progression-free survival according to NED achieved status in the experimental group; (C) overall survival according to treatment groups; (D) overall survival according to NED achieved status in the experimental group. CI, confidence interval; HR, hazard ratio; FOLFOXIRI, 5-fluorouracil, folinic acid, oxaliplatin, and irinotecan; mFOLFOXIRI, modified FOLFOXIRI; NED, no evidence of disease.

(grade 3 or 4$)$ occurred in $46(57.5 \%)$ of 80 patients; the most common events are listed in Table 4. The overall incidence of grade 3 to 4 AEs was slightly higher in the control group $(61.5 \%$ vs. $55.6 \%, \mathrm{P}=0.612)$. The most common toxicity was leukopenia or neutropenia in the two groups: $40.7 \%$ in the experimental group and $42.3 \%$ in the control group. The occurrence of grade 3 or 4 hemorrhage, hypertension, and proteinuria was $1.9 \%, 1.9 \%$, and $1.9 \%$, respectively, in the bevacizumab-containing group, with no significant difference compared with the control group.

\section{Discussion}

The addition of bevacizumab to mFOLFOXIRI chemotherapy in patients with initially technically unresectable CRLMs and $R A S / B R A F / P I K 3 C A$ mutation yielded a better tumor response, and improved survival. The primary end pointrate of clinical NED attained was increased $9.9 \%$ in triplet with bevacizumab compared with triplet alone. Statistical significance was observed in the adjusted $\mathrm{P}$ value after the correction. Additionally, we also observed a significant survival benefit for patients who attained NED, demonstrating that aggressive LATs and NED achieved could be translated into improved survival. This outcome concurred with the findings from two recently published studies in patients with inoperable CRLMs, which also showed that adding a target agent to chemotherapy (with or without a targeted agent) led to improved survival $(8,18)$.

The mFOLFOXIRI plus bevacizumab was designed as a combination regimen in this study to provide chances of conversion for achieving tumor clearance in initially unresectable CRLMs, particularly for patients with $R A S$ / BRAF/PIK3CA mutation. However, the treatment effect on the rate of NED attained in the two groups did not show a statistically significant difference in a preliminary analysis. We observed diffuse LMs $(>10)$ in a higher proportion of patients in the experimental group at baseline. The multivariable logistic model showed significant evidence of benefit from mFOLFOXIRI plus bevacizumab $(\mathrm{P}=0.022)$ taking into account the number of LMs. We surmised that a higher number of LMs made it difficult to eradicate all these lesions completely, and the patients were less likely to 
Table 4 Summary of most common grade $\geq 3$ adverse events

\begin{tabular}{lccc}
\hline Adverse events & mFOLFOXIRI plus bevacizumab $(\mathrm{n}=54), \mathrm{n}(\%)$ & mFOLFOXIRI alone $(\mathrm{n}=26), \mathrm{n}(\%)$ & $\mathrm{P}$ value \\
\hline Total patients & $30(55.6)$ & $16(61.5)$ & 0.612 \\
Leukopenia or neutropenia & $22(40.7)$ & $11(42.3)$ & 0.894 \\
Diarrhea & $2(7.7)$ & $2(3.7)$ & 0.443 \\
Thrombocytopenia & $1(1.9)$ & $2(7.7)$ & 0.245 \\
ALT or AST increased & $5(9.3)$ & $0(0.0)$ & 0.168 \\
Febrile neutropenia & $1(1.9)$ & $2(7.7)$ & 0.245 \\
Fatigue & $0(0.0)$ & $1(3.8)$ & 0.325 \\
Nausea or vomiting & $3(5.6)$ & $1(3.8)$ & 1.000 \\
Intestinal obstructions & $1(1.9)$ & $1(3.8)$ & 0.547 \\
Peripheral neuropathy & $2(3.7)$ & $0(0.0)$ & 1.000 \\
Stomatitis & $1(1.9)$ & $0(0.0)$ & 1.000 \\
Hemorrhage & $1(1.9)$ & $0(0.0)$ & 1.000 \\
Hypertension & $1(1.9)$ & $0(0.0)$ & 1.000 \\
Proteinuria & $1(1.9)$ & $0(0.0)$ & 1.000 \\
\hline
\end{tabular}

ALT, alanine aminotransferase; AST, aspartate aminotransferase; FOLFOXIRI, 5-fluorouracil, folinic acid, oxaliplatin, and irinotecan; mFOLFOXIRI, modified FOLFOXIRI.

attain NED.

Involving triplet chemotherapy has been used as the palliative therapy for mCRC, and has also been tested as the conversion therapy for initially unresectable CRLMs in several studies $(8,11-13,19-21)$ (Table S4). However, very few studies included a chemotherapy-only group. The potential benefits of adding bevacizumab or cetuximab/ panitumumab to triplet chemotherapy backbones in this setting have not been well resolved. Further clinical studies including patients with gene mutation were rare. Recent findings indicated that the $R A S, B R A F$, or PIK3CA mutation did not positively respond to cetuximab/ panitumumab in treating mCRC (14,22-24). Additionally, the presence of mutation in $R A S / B R A F / P I K 3 C A$ was related to worse patient outcomes $(25,26)$. Mutations in these genes were also associated with shorter cancer-specific survival among patients who underwent curative resection of mCRC (27-29). In this study, patients whose tumors harbored one of the aforementioned gene alterations were enrolled; the majority of these carried the $R A S$ mutation. A recent FOCULM study published by us demonstrated that mFOLFOXIRI plus cetuximab was an effective conversion regimen for $R A S / B R A F$ wild-type patients with unresectable CRLMs (8); the long-term survival in the experimental and control groups was superior compared with that in the present study. In this context, we considered that gene mutation might exert a negative impact on patient survival.

In the present study, the $\mathrm{R} 0$ resection and NED attained were observed in $3.8 \%$ and $30.8 \%$ of patients in the mFOLFOXIRI group, respectively. In a phase II METHEP trial, triplet (FOLFIRINOX) facilitated 30\% of patients with initially unresectable LMs to undergo R0 resection (12). Although the R0 resection rate was lower in our study, the mFOLFOXIRI group also achieved a similar percentage of patients with all visible LM lesions eradicated through LAT strategies compared with the METHEP trial. For the long-term efficacy, the median PFS of triplet chemotherapy in this phase II trial was longer than that in our trial (11.9 vs. 9.1 months). Gruenberger et al., in their OLIVIA study, assessed the efficacy of triplet (FOLFOXIRI) or doublet (modified FOLFOX6) plus bevacizumab in this setting (13). In the triplet plus bevacizumab group, the R0 resection rate was $49 \%$, which was higher than $7.4 \%$ of the experimental group in the present study. The median PFS for the triplet plus bevacizumab regimen was higher than that in our study (18.6 vs. 12.6 months). The data showed that the efficacy of conversion therapy in our study might be inferior to that in the METHEP and OLIVIA 
studies. However, several explanations existed for these differences in outcomes. The distribution of the gene status for $R A S / B R A F / P I K 3 C A$ was unknown in the METHEP and OLIVIA studies. Thermal ablation was applied for some patients as the strategy of eradicating LMs in our study. Additionally, a significant proportion of our patients presented with diffuse LMs, which might have a detrimental effect on achieving $\mathrm{R} 0$ resection and long-term survival. The response rate achieved with triplet plus bevacizumab in our study $(77.4 \%)$ was comparable to that reported in the OLIVIA study (81\%), and was higher than that for the same regimen reported in the TRIBE study (65.1\%) (30). A possible explanation was that liver-limited metastatic disease responded better.

The $\mathrm{R} 0$ resection rate in the experimental group in our study was $7.4 \%$, which was lower than $22.3 \%$ of arm A in the BECOME study (18), which enrolled patients with $R A S$ mutant unresectable CRLMs and evaluated the effects of bevacizumab plus mFOLFOX6 (arm A) versus mFOLFOX6 alone ( $\operatorname{arm~B})$ in the first-line setting. This was probably because the majority of patients in our study underwent thermal ablation to remove LMs. Comparing the longterm efficacy of the doublet or triplet plus bevacizumab regimen in the two studies, the median PFS was found to be numerically longer in our study (12.6 vs. 9.5 months). Analogous results were noted in the TRIBE study (26). The median PFS was prolonged for the triplet plus bevacizumab regimen in the $R A S$ and $B R A F$ mutation subgroup, compared with doublet plus bevacizumab. Additionally, the response rate and median PFS in the control group of our study were $60.0 \%$ and 9.1 months, respectively, which were similar to that of $54.5 \%$ and 9.5 months in the BECOME study arm A. Further head-to-head comparison the efficacy of two treatment regimens in prospective studies are warranted.

RAS/BRAF/PIK3CA mutations are associated with worse biology and an aggressive metastasis, making the clinical decision of resection challenging. Studies have shown that ablation permits retreatment of new LMs and recurrences with identical survival to those that had no recurrence (31). In addition, SBRT of LMs has been reported to achieve high local control rates (32). Thus, the rate of NED obtained was chosen as the primary end point in our study, based on the LAT strategies applied under the mode of an MDT. A clear definition about ablation indications and complete ablation, as described in our previous study (8), was also set to improve local tumor control. In the present study, 6 and 17 patients underwent R0 resection plus thermal ablation and complete thermal ablation, respectively, and one patient in the experimental group underwent $\mathrm{R} 0$ resection plus complete thermal ablation and SBRT. We observed that thermal ablation yielded a local tumor control rate close to surgical resection (Table S5). Additionally, a significant difference was observed for OS among patients who received systemic therapy plus complete ablation compared with those who received systemic therapy alone (no LAT) (Figure S2), which was consistent with the findings of other studies $(10,33)$.

The observed safety profiles were generally consistent with those in previous studies involving triplet chemotherapy $(8,11,34)$. The main grade 3 or 4 AEs of the modified triplet chemotherapy in this study were leukopenia/neutropenia and diarrhea, but a relatively higher percentage of patients with thrombocytopenia was identified in the control group. Overall, this modified triplet chemotherapy remains feasible, and early recognition and active management of AEs is critical. Potential AEs associated with bevacizumab include hemorrhage, gastrointestinal perforation, anastomotic leakage, hypertension, proteinuria, thrombosis, and wound-healing complications that can interfere with the treatment process $(18,35)$. In the experimental group of our study, bevacizumab was interrupted 6-8 weeks before all surgeries and restarted if the wound was completely healed. Besides, patients had discontinued bevacizumab two weeks before thermal ablation. One patient (in the experimental group) had grade 3 hemorrhage after undergoing radical colectomy. Of the 16 patients who underwent hepatectomy (in the experimental group), no grade 3 or 4 hemorrhage, anastomotic leakage, or wound healing was observed in the perioperative period. The percentage of bevacizumab-related AEs (grade $\geq 3$ ) was low in this study, which might be ascribed to the well aware of the instructions for bevacizumab by oncologists. Moreover, it was suggested that bevacizumab did not affect the safety profile of the modified triplet chemotherapy in this study.

The results should be interpreted with caution for several weaknesses in our study. First, this was a singlecenter study enrolling a small number of patients produced a limited level of evidence. Second, patients were not randomly assigned but were grouped into two groups based on their choice, resulting in some unmeasured confounders. Third, tumor response was not assessed by a blinded independent radiologic review, leading to bias in the results. Moreover, our analysis did not take into account potential differences among different gene mutations. However, despite these limitations, the present phase II study was novel in prospectively assessing bevacizumab combined 
with mFOLFOXIRI compared with mFOLFOXIRI alone as conversion therapy among patients with the RAS/BRAF/ PIK3CA mutant CRLMs. Together with the favorable efficacy and safety data from our study, mFOLFOXIRIbased chemotherapy regimen, should be used as upfront treatment in patients with initially unresectable disease with conversion intent and those with good PS with palliative intent.

\section{Conclusions}

Our study showed that adding bevacizumab to mFOLFOXIRI in patients with the initially unresectable RAS/BRAF/PIK3CA mutant CRLMs increased the rate of NED obtained with an acceptable safety profile and showed a trend toward improved survival. Further randomized trials should be conducted to define better the relative benefits of the mFOLFOXIRI plus bevacizumab regimen in this setting.

\section{Acknowledgments}

The authors would like to thank all patients and their families for participating in this study.

Funding: This work was supported by the National Key Research and Development Program of China (grant number 2019YFC1316003) and National Natural Science Foundation of China (grant number 81974369).

\section{Footnote}

Reporting Checklist: The authors have completed the TREND reporting checklist. Available at https://atm. amegroups.com/article/view/10.21037/atm-21-6731/rc

Data Sharing Statement: Available at https://atm.amegroups. com/article/view/10.21037/atm-21-6731/dss

Conflicts of Interest: All authors have completed the ICMJE uniform disclosure form (available at https://atm. amegroups.com/article/view/10.21037/atm-21-6731/coif). The authors have no conflicts of interest to declare.

Ethical Statement: The authors are accountable for all aspects of the work in ensuring that questions related to the accuracy or integrity of any part of the work are appropriately investigated and resolved. The trial was conducted in accordance with the Declaration of Helsinki (as revised in 2013). This study was approved by ethics committee of The Sixth Affiliated Hospital, Sun Yat-sen University (No. 2014ZSLYEC-024) and informed consent was taken from all individual participants.

Open Access Statement: This is an Open Access article distributed in accordance with the Creative Commons Attribution-NonCommercial-NoDerivs 4.0 International License (CC BY-NC-ND 4.0), which permits the noncommercial replication and distribution of the article with the strict proviso that no changes or edits are made and the original work is properly cited (including links to both the formal publication through the relevant DOI and the license). See: https://creativecommons.org/licenses/by-nc-nd/4.0/.

\section{References}

1. Maeda Y, Shinohara T, Minagawa N, et al. Oncological outcomes of repeat metastasectomy for recurrence after hepatectomy for colorectal liver metastases. A case series. Ann Med Surg (Lond) 2020;52:24-30.

2. Adam R, Haller DG, Poston G, et al. Toward optimized front-line therapeutic strategies in patients with metastatic colorectal cancer--an expert review from the International Congress on Anti-Cancer Treatment (ICACT) 2009. Ann Oncol 2010;21:1579-84.

3. Kemeny NE, Melendez FD, Capanu M, et al. Conversion to resectability using hepatic artery infusion plus systemic chemotherapy for the treatment of unresectable liver metastases from colorectal carcinoma. J Clin Oncol 2009;27:3465-71.

4. Folprecht G, Martinelli E, Mazard T, et al. Triplet chemotherapy in combination with anti-EGFR agents for the treatment of metastatic colorectal cancer: Current evidence, advances, and future perspectives. Cancer Treat Rev 2022;102:102301.

5. Folprecht G, Gruenberger T, Bechstein WO, et al. Tumour response and secondary resectability of colorectal liver metastases following neoadjuvant chemotherapy with cetuximab: the CELIM randomised phase 2 trial. Lancet Oncol 2010;11:38-47.

6. Yasuno $M$, Uetake $H$, Ishiguro $M$, et al. mFOLFOX6 plus bevacizumab to treat liver-only metastases of colorectal cancer that are unsuitable for upfront resection (TRICC0808): a multicenter phase II trial comprising the final analysis for survival. Int J Clin Oncol 2019;24:516-25.

7. Van Cutsem E, Cervantes A, Adam R, et al. ESMO consensus guidelines for the management of patients with metastatic colorectal cancer. Ann Oncol 
2016;27:1386-422.

8. Hu H, Wang K, Huang M, et al. Modified FOLFOXIRI With or Without Cetuximab as Conversion Therapy in Patients with RAS/BRAF Wild-Type Unresectable Liver Metastases Colorectal Cancer: The FOCULM Multicenter Phase II Trial. Oncologist 2021;26:e90-8.

9. Strobel O, Büchler MW. Unresectable colorectal liver metastases : Prolonged survival with aggressive local therapy. Chirurg 2017;88:803.

10. Ruers T, Van Coevorden F, Punt CJ, et al. Local Treatment of Unresectable Colorectal Liver Metastases: Results of a Randomized Phase II Trial. J Natl Cancer Inst 2017;109:djx015.

11. Falcone A, Ricci S, Brunetti I, et al. Phase III trial of infusional fluorouracil, leucovorin, oxaliplatin, and irinotecan (FOLFOXIRI) compared with infusional fluorouracil, leucovorin, and irinotecan (FOLFIRI) as firstline treatment for metastatic colorectal cancer: the Gruppo Oncologico Nord Ovest. J Clin Oncol 2007;25:1670-6.

12. Ychou M, Rivoire M, Thezenas S, et al. A randomized phase II trial of three intensified chemotherapy regimens in first-line treatment of colorectal cancer patients with initially unresectable or not optimally resectable liver metastases. The METHEP trial. Ann Surg Oncol 2013;20:4289-97.

13. Gruenberger T, Bridgewater J, Chau I, et al. Bevacizumab plus mFOLFOX-6 or FOLFOXIRI in patients with initially unresectable liver metastases from colorectal cancer: the OLIVIA multinational randomised phase II trial. Ann Oncol 2015;26:702-8.

14. De Roock W, Claes B, Bernasconi D, et al. Effects of KRAS, BRAF, NRAS, and PIK3CA mutations on the efficacy of cetuximab plus chemotherapy in chemotherapyrefractory metastatic colorectal cancer: a retrospective consortium analysis. Lancet Oncol 2010;11:753-62.

15. Vakiani E, Janakiraman $M$, Shen $R$, et al. Comparative genomic analysis of primary versus metastatic colorectal carcinomas. J Clin Oncol 2012;30:2956-62.

16. Deng Y; Chinese Southwest Oncology Group - the Committee of Colorectal Cancer. The "Chinese Expert Consensus on the Clinical Application of the Chinese Modified Triplet Combination with Irinotecan (CPT-11), Oxaliplatin (LOHP), Continuous Infusion 5-Fluorouracil, and Leucovorin for Colorectal Cancer". Gastroenterol Rep (Oxf) 2021;9:279-89.

17. Pawlik TM, Scoggins CR, Zorzi D, et al. Effect of surgical margin status on survival and site of recurrence after hepatic resection for colorectal metastases. Ann Surg
2005;241:715-22, discussion 722-4.

18. Tang W, Ren L, Liu T, et al. Bevacizumab Plus mFOLFOX6 Versus mFOLFOX6 Alone as First-Line Treatment for RAS Mutant Unresectable Colorectal Liver-Limited Metastases: The BECOME Randomized Controlled Trial. J Clin Oncol 2020;38:3175-84.

19. Ychou M, Viret F, Kramar A, et al. Tritherapy with fluorouracil/leucovorin, irinotecan and oxaliplatin (FOLFIRINOX): a phase II study in colorectal cancer patients with non-resectable liver metastases. Cancer Chemother Pharmacol 2008;62:195-201.

20. Masi G, Loupakis F, Salvatore L, et al. Bevacizumab with FOLFOXIRI (irinotecan, oxaliplatin, fluorouracil, and folinate) as first-line treatment for metastatic colorectal cancer: a phase 2 trial. Lancet Oncol 2010;11:845-52.

21. Garufi C, Torsello A, Tumolo S, et al. Cetuximab plus chronomodulated irinotecan, 5-fluorouracil, leucovorin and oxaliplatin as neoadjuvant chemotherapy in colorectal liver metastases: POCHER trial. Br J Cancer 2010;103:1542-7.

22. Laurent-Puig P, Cayre A, Manceau G, et al. Analysis of PTEN, BRAF, and EGFR status in determining benefit from cetuximab therapy in wild-type KRAS metastatic colon cancer. J Clin Oncol 2009;27:5924-30.

23. Therkildsen C, Bergmann TK, Henrichsen-Schnack T, et al. The predictive value of KRAS, NRAS, BRAF, PIK3CA and PTEN for anti-EGFR treatment in metastatic colorectal cancer: A systematic review and meta-analysis. Acta Oncol 2014;53:852-64.

24. Goel G. Molecular characterization and biomarker identification in colorectal cancer: Toward realization of the precision medicine dream. Cancer Manag Res 2018;10:5895-908.

25. Cathomas G. PIK3CA in Colorectal Cancer. Front Oncol 2014;4:35.

26. Cremolini C, Loupakis F, Antoniotti C, et al. FOLFOXIRI plus bevacizumab versus FOLFIRI plus bevacizumab as first-line treatment of patients with metastatic colorectal cancer: updated overall survival and molecular subgroup analyses of the open-label, phase 3 TRIBE study. Lancet Oncol 2015;16:1306-15.

27. Ogino S, Nosho K, Kirkner GJ, et al. PIK3CA mutation is associated with poor prognosis among patients with curatively resected colon cancer. J Clin Oncol 2009;27:1477-84.

28. Tosi F, Magni E, Amatu A, et al. Effect of KRAS and BRAF Mutations on Survival of Metastatic Colorectal Cancer After Liver Resection: A Systematic Review and 
Meta-Analysis. Clin Colorectal Cancer 2017;16:e153-63.

29. Brunsell TH, Sveen A, Bjørnbeth BA, et al. High Concordance and Negative Prognostic Impact of RAS/ BRAF/PIK3CA Mutations in Multiple Resected Colorectal Liver Metastases. Clin Colorectal Cancer 2020;19:e26-47.

30. Loupakis F, Cremolini C, Masi G, et al. Initial therapy with FOLFOXIRI and bevacizumab for metastatic colorectal cancer. N Engl J Med 2014;371:1609-18.

31. Ricke J, Mohnike K, Pech M, et al. Local response and impact on survival after local ablation of liver metastases from colorectal carcinoma by computed tomographyguided high-dose-rate brachytherapy. Int J Radiat Oncol Biol Phys 2010;78:479-85.

32. Barry A, Wong R, Dawson LA. The Management of Colorectal Cancer Liver Metastases: The Radiation
Oncology Viewpoint. Int J Radiat Oncol Biol Phys 2019;103:540-1.

33. Ruers T, Punt C, Van Coevorden F, et al. Radiofrequency ablation combined with systemic treatment versus systemic treatment alone in patients with non-resectable colorectal liver metastases: a randomized EORTC Intergroup phase II study (EORTC 40004). Ann Oncol 2012;23:2619-26.

34. Modest DP, Martens UM, Riera-Knorrenschild J, et al. FOLFOXIRI Plus Panitumumab As First-Line Treatment of RAS Wild-Type Metastatic Colorectal Cancer: The Randomized, Open-Label, Phase II VOLFI Study (AIO KRK0109). J Clin Oncol 2019;37:3401-11.

35. Sun W. Angiogenesis in metastatic colorectal cancer and the benefits of targeted therapy. J Hematol Oncol 2012;5:63.
Cite this article as: Shen $\mathrm{C}, \mathrm{Hu} \mathrm{H}$, Cai Y, Ling J, Zhang J, Wu Z, Xie X, Huang M, Wang H, Kang L, Lan P, Wu X, Liu G, Wan Y, Zhou Z, Huang Y, Li F, Wang H, Ma T, Luo S, Cai Y, Shi L, Deng Y. mFOLFOXIRI with or without bevacizumab for conversion therapy of $R A S / B R A F / P I K 3 C A$ mutant unresectable colorectal liver metastases: the FORBES non-randomized phase II trial. Ann Transl Med 2022;10(4):171. doi: 10.21037/atm-216731 


\section{Supplementary}

Table S1 Twenty-six patients were recruited into the control group for the following reasons

\begin{tabular}{lc}
\hline Reasons & N \\
\hline Poor financial status & 24 \\
Previous myocardial infarction & 1 \\
Grade 3 hypertension & 1 \\
\hline
\end{tabular}

Table S2 Number of cycles and relative dose intensities of mFOLFOXIRI regimen, according to treatment group

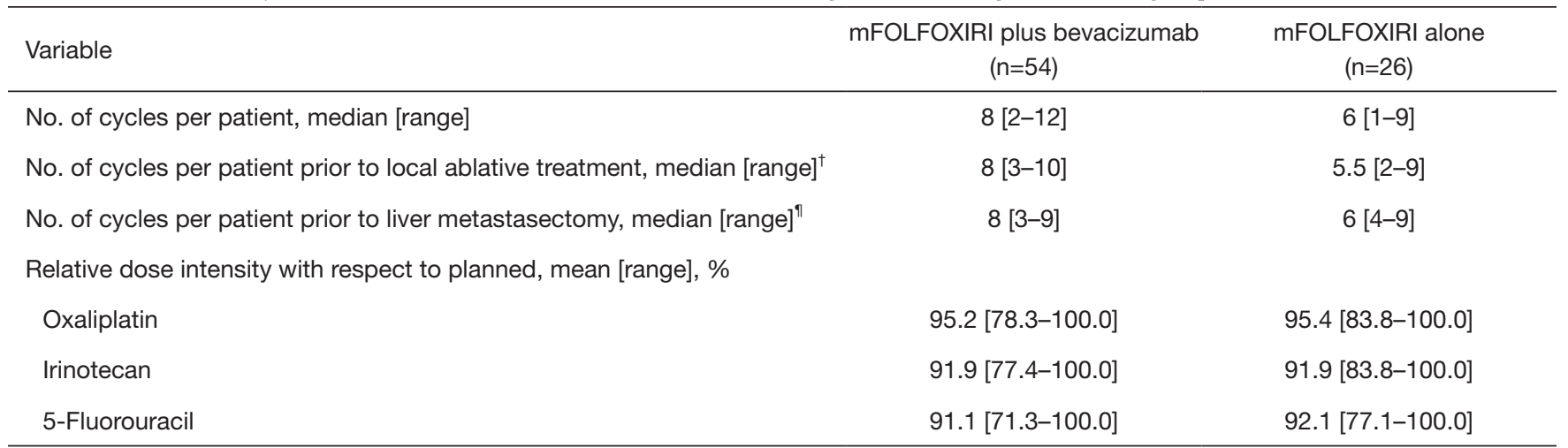

${ }^{\dagger}$, only patients received local ablative treatment; ", only patients received liver metastasectomy. FOLFOXIRI, 5-fluorouracil, folinic acid, oxaliplatin, and irinotecan; mFOLFOXIRI, modified FOLFOXIRI.

Table S3 Second line therapy for patients with disease progression, according to treatment group

\begin{tabular}{lcc}
\hline \multirow{2}{*}{ Regimen } & \multicolumn{1}{c}{$\mathrm{N}(\%)^{\dagger}$} & \\
\cline { 2 - 3 } & mFOLFOXIRI plus bevacizumab $(\mathrm{n}=33)$ & mFOLFOXIRI alone $(\mathrm{n}=12)$ \\
\hline Triplet chemotherapy plus bevacizumab & $24(72.7)$ & $3(25.0)$ \\
Doublet chemotherapy plus bevacizumab & $2(6.1)$ & $6(50.0)$ \\
Doublet chemotherapy & $0(0)$ & $1(8.3)$ \\
Capecitabine & $2(6.1)$ & $0(0)$ \\
Regorafenib plus PD-1 inhibitor & $0(0)$ & $1(8.3)$ \\
Fruquintinib & & \\
\hline
\end{tabular}

Percentages may not total 100 because of rounding. ${ }^{\dagger}$, only patients receiving second line therapy were included. FOLFOXIRI, 5 -fluorouracil, folinic acid, oxaliplatin, and irinotecan; mFOLFOXIRI, modified FOLFOXIRI; PD-1, programmed death-1. 

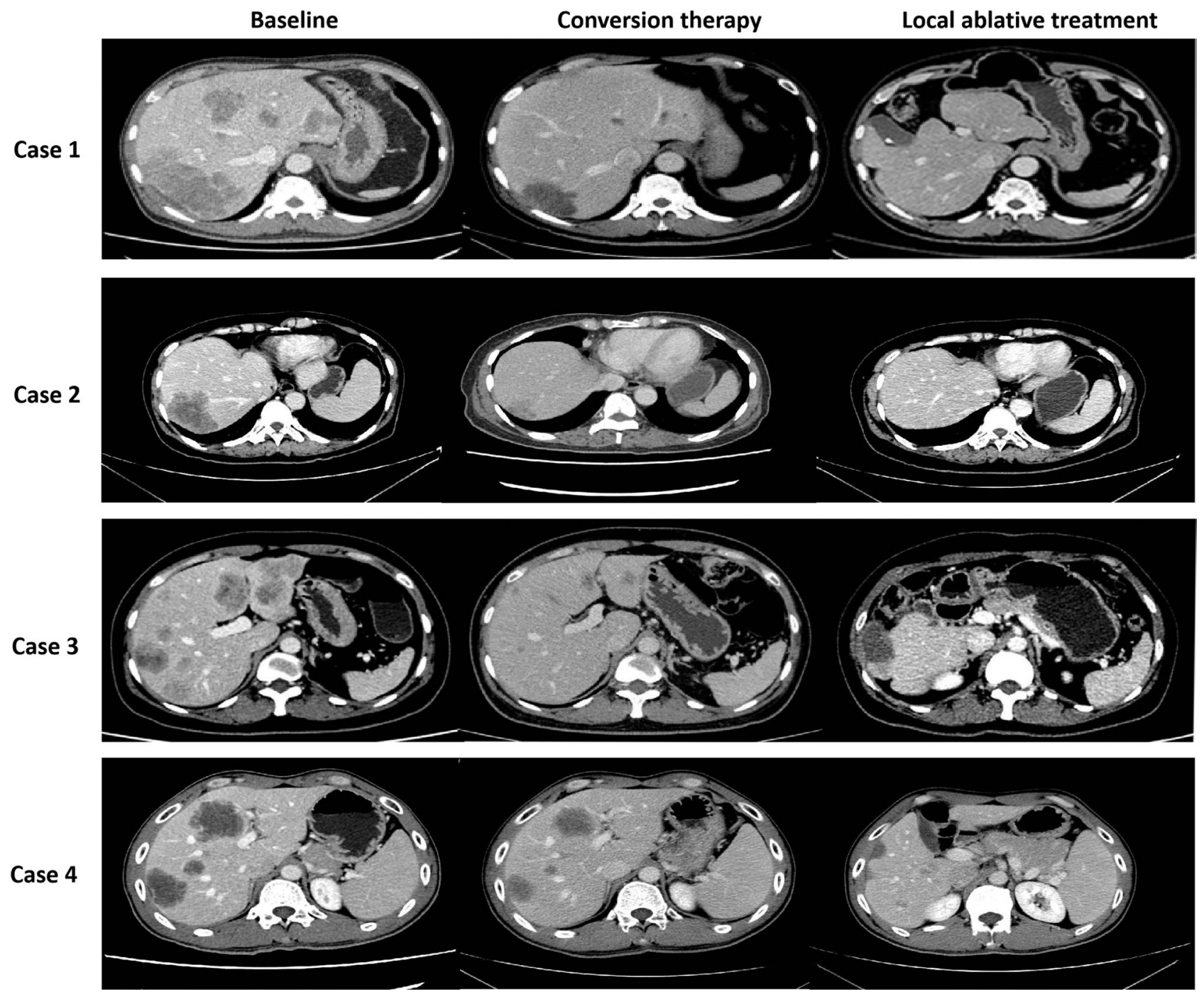

Figure S1 Radiographic images of four cases before and after treatment in the two groups. Case 1: the patient in the experimental group attained NED; Case 2 (only the largest lesions was shown): the patient in the control group attained NED; Case 3: the patient in the experimental group did not attain NED; Case 4: the patient in the control group did not attain NED. NED, no evidence of disease. 
Table S4 Clinical studies involving triplet chemotherapy-based regimen as conversion therapy for patients with initially unresectable CRLMs

\begin{tabular}{|c|c|c|c|c|c|c|}
\hline Schedule & Gene status & $\mathrm{N}$ & $\begin{array}{l}\mathrm{RR} \\
(\%)\end{array}$ & $\begin{array}{l}\text { R0 resection } \\
(\%)\end{array}$ & $\begin{array}{c}\text { mPFS } \\
\text { (months) }\end{array}$ & $\begin{array}{c}\mathrm{mOS} \\
\text { (months) }\end{array}$ \\
\hline FOLFOXIRI (Falcone et al., 2007) (11) & Unknown & 39 & Not reported & 36 & Not reported & 23.4 \\
\hline FOLFIRINOX (Ychou et al., 2008) (19) & Unknown & 34 & 70.6 & 26.5 & Not reported & 36 \\
\hline $\begin{array}{l}\text { FOLFOXIRI + bevacizumab } \\
\text { (Masi et al., 2010) (20) }\end{array}$ & Unknown & 30 & 80 & 40 & 16.9 & Not reported \\
\hline $\begin{array}{l}\text { FOLFOXIRI + cetuximab } \\
\text { (Garufi et al., 2010) (21) }\end{array}$ & Unknown & 43 & 79.1 & 60 & 14 & 37 \\
\hline FOLFIRINOX (Ychou et al., 2013) (12) & Unknown & 30 & 73 & 30 & 11.9 & Not reported \\
\hline $\begin{array}{l}\text { FOLFOXIRI + bevacizumab } \\
\text { (Gruenberger et al., 2015) (13) }\end{array}$ & Unknown & 41 & 81 & 49 & 18.6 & Not reached \\
\hline mFOLFOXIRI (Hu et al., 2021) (8) & $R A S / B R A F$ wild-type & 34 & 76.5 & 20.6 & 14.2 & 33.2 \\
\hline $\begin{array}{l}\text { mFOLFOXIRI + cetuximab } \\
\text { (Hu et al., 2021) (8) }\end{array}$ & $R A S / B R A F$ wild-type & 67 & 95.5 & 35.8 & 15.5 & Not reached \\
\hline $\begin{array}{l}\text { mFOLFOXIRI } \\
\text { (Shen et al., present study) }\end{array}$ & RAS/BRAF/PIK3CA mutation & 26 & 60.0 & 3.8 & 9.1 & 35.3 \\
\hline $\begin{array}{l}\text { mFOLFOXIRI+ bevacizumab } \\
\text { (Shen et al., present study) }\end{array}$ & RAS/BRAF/PIK3CA mutation & 54 & 77.4 & 7.4 & 12.6 & 42.6 \\
\hline
\end{tabular}

CRLMs, colorectal liver-limited metastases; FOLFOXIRI, 5-fluorouracil, folinic acid, oxaliplatin, and irinotecan; FOLFIRINOX, fluorouracil, leucovorin, oxaliplatin, and irinotecan; mFOLFOXIRI, modified FOLFOXIRI; mOS, median overall survival; mPFS, median progression-free survival; RR, response rate.

Table S5 Site of first disease progression among patients who underwent LATs, according to treatment group

\begin{tabular}{|c|c|c|}
\hline \multirow{2}{*}{ LATs and site of first progression } & \multicolumn{2}{|c|}{$\mathrm{N}(\%)$} \\
\hline & mFOLFOXIRI plus bevacizumab $(n=32)$ & mFOLFOXIRI alone $(n=16)$ \\
\hline $\mathrm{R} 0$ resection plus thermal ablation & $11^{\dagger}$ & 1 \\
\hline Site treated by surgical resection & $1(9.1)$ & $0(0.0)$ \\
\hline Site treated by thermal ablation & $0(0.0)$ & $0(0.0)$ \\
\hline Any other liver lesions & $5(45.5)$ & $0(0.0)$ \\
\hline Extrahepatic lesions only & $2(18.2)$ & $0(0.0)$ \\
\hline Without disease progression & $3(27.3)$ & $1(100.0)$ \\
\hline Complete thermal ablation & 10 & 7 \\
\hline Site treated by thermal ablation & $1(10.0)$ & $1(14.3)$ \\
\hline Any other liver lesions & $4(40.0)$ & $1(14.3)$ \\
\hline Extrahepatic lesions & $1(10.0)$ & $2(28.6)$ \\
\hline Without disease progression & $4(40.0)$ & $3(42.9)$ \\
\hline Thermal ablation & 8 & 4 \\
\hline Site treated by thermal ablation & $0(0.0)$ & $0(0.0)$ \\
\hline Any other liver lesions & $4(50.0)$ & $4(100.0)$ \\
\hline Extrahepatic lesions & $1(12.5)$ & $0(0.0)$ \\
\hline Without disease progression & $3(37.5)$ & $0(0.0)$ \\
\hline $\mathrm{R} 2$ resection & 3 & $4^{\pi}$ \\
\hline Site treated by surgical resection & $0(0.0)$ & $0(0.0)$ \\
\hline Any other liver lesions & $3(100.0)$ & $2(50.0)$ \\
\hline Extrahepatic lesions & $0(0.0)$ & $0(0.0)$ \\
\hline Without disease progression & $0(0.0)$ & $2(50.0)$ \\
\hline
\end{tabular}

${ }^{\dagger}$, one patient underwent $\mathrm{R} 0$ resection plus thermal ablation and stereotactic body radiation therapy; ", one patient underwent R2 resection plus thermal ablation and transarterial chemoembolisation. FOLFOXIRI, 5-fluorouracil, folinic acid, oxaliplatin, and irinotecan; LATs, local ablative treatments; mFOLFOXIRI, modified FOLFOXIRI. 


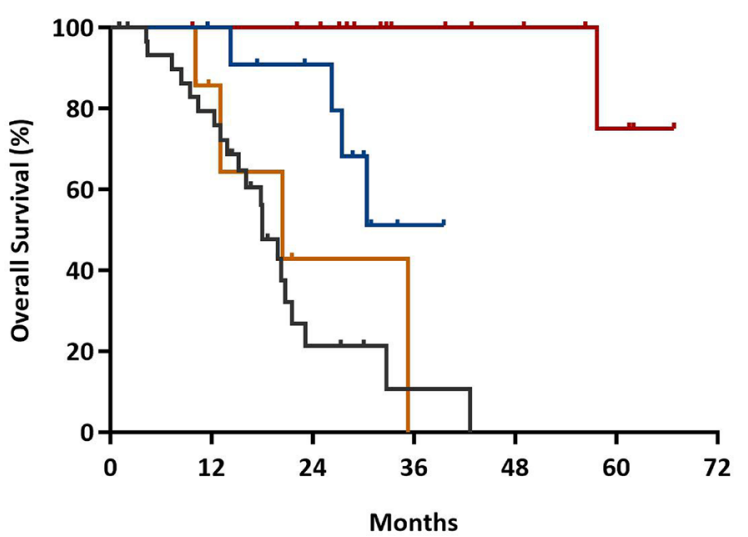

- Complete thermal ablation

$\rightarrow \mathrm{R} 0$ resection \pm thermal ablation

- R2 resection

- No LAT

Complete thermal ablation vs. No LAT: $\mathrm{HR}, 0.009$ (95\% Cl, 0.000-0.326) $\mathrm{P}<0.001$

R0 resection \pm thermal ablation vs. No LAT:

$\mathrm{HR}, 0.233(95 \% \mathrm{Cl}, 0.078-0.700) \mathrm{P}=0.005$

R2 resection vs. No LAT:

HR, $0.722(95 \% \mathrm{Cl}, 0.243-2.149) \mathrm{P}=0.558$

Figure S2 Kaplan-Meier curves of overall survival, according to means of LAT. CI, confidence interval; HR, hazard ratio; LAT, local ablative treatment. 